\title{
Exact Solutions of Fractional Burgers and Cahn-Hilliard Equations Using Extended Fractional Riccati Expansion Method
}

\author{
Wei Li, Huizhang Yang, and Bin He \\ College of Mathematics of Honghe University, Mengzi, Yunnan 661100, China \\ Correspondence should be addressed to Wei Li; wellars@163.com
}

Received 13 February 2014; Accepted 18 May 2014; Published 10 June 2014

Academic Editor: Miguel A. F. Sanjuan

Copyright ( 2014 Wei Li et al. This is an open access article distributed under the Creative Commons Attribution License, which permits unrestricted use, distribution, and reproduction in any medium, provided the original work is properly cited.

\begin{abstract}
Based on a general fractional Riccati equation and with Jumarie's modified Riemann-Liouville derivative to an extended fractional Riccati expansion method for solving the time fractional Burgers equation and the space-time fractional Cahn-Hilliard equation, the exact solutions expressed by the hyperbolic functions and trigonometric functions are obtained. The obtained results show that the presented method is effective and appropriate for solving nonlinear fractional differential equations.
\end{abstract}

\section{Introduction}

During recent years, fractional differential equations (FDEs) have attracted much attention because of their potential applications in engineering and applied sciences such as signal processing, materials and mechanics, biology systems, anomalous diffusion, and medical [1-4]. Thus, it is very important to find some proper methods for solving fractional differential equations. Many effective methods have been established to obtain the solutions of FDEs, such as the variational iteration method $[5,6]$, the finite difference method [7], the fractional complex transform $[8,9]$, the exponential function method [10], the fractional subequation method [11], the $\left(G^{\prime} / G\right)$-expansion method $[12,13]$, and the first integral method [14]. However, the solutions of most fractional differential equations cannot be obtained explicitly in general. As a consequence, it is necessary to extend the existing methods for the fractional differential equations.

Recently, based on Jumarie's modified Riemann-Liouville derivative and the fractional Riccati equation $D_{\xi}^{\alpha} \phi(\xi)=$ $\sigma+\phi^{2}(\xi)$, S. Zhang and H.-Q. Zhang [15] first proposed a new direct method called fractional subequation method in solving nonlinear time fractional biological population model and (4+1)-dimensional space-time fractional Fokas equation. Soon, the method was further improved by Guo et al. [16] and they obtained the analytical solutions of the space-time fractional Whitham-Broer-Kaup and generalized
Hirota-Satsuma coupled KdV equations by introducing a new general ansatz. In a similar way, Lu [17] modified the method to derive the rational formal solutions of the spacetime fractional Whitham-Broer-Kaup, the foam drainage equation with time and space-fractional derivatives, and nonlinear time fractional biological population model. Very lately, by extending the fractional Riccati equation in [1517] to the more general form $D_{\xi}^{\alpha} F(\xi)=A+B F^{2}(\xi)$, Abdel-Salam and Yousif [18] presented the fractional Riccati expansion method to obtain exact solutions of the space-time fractional Korteweg-de Vries equation, the spacetime fractional RLW equation, the space-time fractional Boussinesq equation, and the space-time fractional KleinGordon equation. In this paper, based on Jumarie's modified Riemann-Liouville derivative and the general fractional Riccati equation $D_{\xi}^{\alpha} F(\xi)=A+B F(\xi)+C F^{2}(\xi)$, we will introduce an extended fractional Riccati expansion method to the time fractional Burgers equation and the space-time fractional Cahn-Hilliard equation [19]

$$
\begin{gathered}
\frac{\partial^{\alpha} u}{\partial t^{\alpha}}+\varepsilon u \frac{\partial u}{\partial x}-\nu \frac{\partial^{2} u}{\partial x^{2}}=0, \quad t>0,0<\alpha \leq 1, \\
D_{t}^{\alpha} u-\gamma D_{x}^{\alpha} u-6 u\left(D_{x}^{\alpha} u\right)^{2}-\left(3 u^{2}-1\right) D_{x}^{2 \alpha} u+D_{x}^{4 \alpha} u=0, \\
0<\alpha \leq 1,
\end{gathered}
$$


to construct new exact solutions. This paper is organized as follows. In Section 2, some basic properties of Jumarie's modified Riemann-Liouville derivative are given. In Section 3, the main steps of the extended fractional Riccati expansion method are given. In Sections 4 and 5, we construct the exact solutions of (1) and (2) by the proposed method. Some conclusions are given in Section 6.

\section{Preliminaries}

There are several definitions for fractional differential equations. These definitions include Caputo's fractional derivative [20], Kolwankar-Gangal derivative [21], and Jumarie's modified Riemann-Liouville derivative $[22,23]$. In this, we give the modified Riemann-Liouville derivative defined by Jumarie:

$$
\begin{array}{r}
D_{x}^{\alpha} f(x)=\frac{1}{\Gamma(1-\alpha)} \frac{d}{d x} \int_{0}^{x}(x-\xi)^{-\alpha}[f(\xi)-f(0)] d \xi, \\
0<\alpha<1, \\
D_{x}^{\alpha} f(x)=\left(f^{(n)}(x)\right)^{(\alpha-n)}, \quad n \leq \alpha \leq n+1, n \geq 1,
\end{array}
$$

where $f: R \rightarrow R, x \rightarrow f(x)$ denote a continuous (but not necessarily differentiable) function. Some useful formulas of Jumarie's modified Riemann-Liouville derivative were summarized in $[22,23]$; three of them are

$$
\begin{gathered}
D_{x}^{\alpha} x^{\gamma}=\frac{\Gamma(1+\gamma)}{\Gamma(1+\gamma-\alpha)} x^{\gamma-\alpha}, \quad \gamma>0, \\
D_{x}^{\alpha}(u(x) v(x))=v(x) D_{x}^{\alpha} u(x)+u(x) D_{x}^{\alpha} v(x), \\
D_{x}^{\alpha} f[u(x)]=f_{u}^{\prime}[u(x)] D_{x}^{\alpha} u(x)=D_{u}^{\alpha} f[u(x)]\left(u^{\prime}(x)\right)^{\alpha},
\end{gathered}
$$

which will be used in the following sections.

\section{Extended Fractional Riccati Expansion Method}

In this section, we give the description of the extended fractional Riccati expansion method for solving the nonlinear FDE as

$$
P\left(u, u_{t}, u_{x}, D_{t}^{\alpha} u, D_{x}^{\alpha} u, \ldots\right)=0, \quad 0<\alpha \leq 1,
$$

where $u$ is an unknown function and $P$ is a polynomial of $u$ and its various partial derivatives, in which the highest order derivatives and nonlinear terms are involved.

Step 1. Using the traveling wave transformation

$$
u(x, t)=U(\xi), \quad \xi=x+\omega t,
$$

where $\omega$ is a constant to be determined later, the nonlinear FDE (5) is reduced to a nonlinear fractional ordinary differential equation

$$
Q\left(U(\xi), U^{\prime}(\xi), U^{\prime \prime}(\xi), D_{\xi}^{\alpha} U(\xi), \ldots\right)=0,
$$

where ${ }^{\text {' } "}=d / d \xi$.
Step 2. Suppose that the solution of ODE (7) can be written as follows:

$$
U=a_{0}+\sum_{j=1}^{n}\left(a_{j} F^{j}(\xi)+b_{j} F^{-j}(\xi)\right),
$$

where $a_{0}, a_{j}, b_{j}(j=1,2, \ldots, n)$ are constants to be determined later and $n$ is a positive integer that is given by the homogeneous balance principle, and $F=F(\xi)$ satisfies the Riccati equation

$$
D_{\xi}^{\alpha} F(\xi)=A+B F(\xi)+C F^{2}(\xi), \quad 0<\alpha \leq 1,
$$

where $A, B$, and $C$ are constants. Using the Mittag-Leffler function in one parameter $E_{\alpha}(x)=\sum_{k=0}^{\infty}\left(x^{k} / \Gamma(1+k \alpha)\right)$, $(\alpha>0)$, we obtain the following solutions of (9).

Type 1. When $B=0$ and $A / C<0$, the solutions of (9) are

$$
\begin{gathered}
F(\xi)=-\sqrt{-\frac{A}{C}} \tanh _{\alpha}\left(C \sqrt{-\frac{A}{C}} \xi\right) \\
F(\xi)=-\sqrt{-\frac{A}{C}} \operatorname{coth}_{\alpha}\left(C \sqrt{-\frac{A}{C}} \xi\right) \\
F(\xi)=-\sqrt{-\frac{A}{C}} \\
\times\left[\operatorname{coth}_{\alpha}\left(2 C \sqrt{-\frac{A}{C}} \xi\right) \pm \operatorname{csch}_{\alpha}\left(2 C \sqrt{-\frac{A}{C}} \xi\right)\right] .
\end{gathered}
$$

When $B=0$ and $A / C>0$, the solutions of (9) are

$$
\begin{gathered}
F(\xi)=\sqrt{\frac{A}{C}} \tan _{\alpha}\left(C \sqrt{\frac{A}{C}} \xi\right), \\
F(\xi)=-\sqrt{\frac{A}{C}} \cot _{\alpha}\left(C \sqrt{\frac{A}{C}} \xi\right) \\
F(\xi)=\sqrt{\frac{A}{C}}\left[\tan _{\alpha}\left(2 C \sqrt{\frac{A}{C}} \xi\right) \pm \sec _{\alpha}\left(2 C \sqrt{\frac{A}{C}} \xi\right)\right] \\
F(\xi)=-\sqrt{\frac{A}{C}}\left[\cot _{\alpha}\left(2 C \sqrt{\frac{A}{C}} \xi\right) \pm \csc _{\alpha}\left(2 C \sqrt{\frac{A}{C}} \xi\right)\right] .
\end{gathered}
$$

Type 2. When $A=0$ and $B C \neq 0$, the solution of (9) is

$$
F(\xi)=-\frac{B d}{C\left[d+\cosh _{\alpha}(B \xi)-\sinh _{\alpha}(B d)\right]},
$$

where $d$ is an arbitrary constant.

Type 3. When $A B C \neq 0$ and $\Delta=B^{2}-4 A C>0$, the solutions of (9) are

$$
\begin{gathered}
F(\xi)=\frac{2 A \cosh _{\alpha}((\sqrt{\Delta} / 2) \xi)}{\sqrt{\Delta} \sinh _{\alpha}((\sqrt{\Delta} / 2) \xi)-B \cosh _{\alpha}((\sqrt{\Delta} / 2) \xi)}, \\
F(\xi)=-\frac{2 A \sinh _{\alpha}((\sqrt{\Delta} / 2) \xi)}{B \sinh _{\alpha}((\sqrt{\Delta} / 2) \xi)-\sqrt{\Delta} \cosh _{\alpha}((\sqrt{\Delta} / 2) \xi)} .
\end{gathered}
$$


When $A B C \neq 0$ and $\Delta=B^{2}-4 A C<0$, the solutions of (9) are

$$
\begin{gathered}
F(\xi)=-\frac{2 A \cos _{\alpha}((\sqrt{-\Delta} / 2) \xi)}{\sqrt{-\Delta} \sin _{\alpha}((\sqrt{-\Delta} / 2) \xi)+B \cos _{\alpha}((\sqrt{-\Delta} / 2) \xi)}, \\
F(\xi)=-\frac{2 A \sin _{\alpha}((\sqrt{-\Delta} / 2) \xi)}{B \sin _{\alpha}((\sqrt{-\Delta} / 2) \xi)-\sqrt{-\Delta} \cos _{\alpha}((\sqrt{-\Delta} / 2) \xi)} .
\end{gathered}
$$

The generalized hyperbolic and trigonometric functions in the above solutions (10)-(21) are defined as

$$
\begin{aligned}
\sinh _{\alpha}(\xi) & =\frac{E_{\alpha}\left(\xi^{\alpha}\right)-E_{\alpha}\left(-\xi^{\alpha}\right)}{2}, \\
\cosh _{\alpha}(\xi) & =\frac{E_{\alpha}\left(\xi^{\alpha}\right)+E_{\alpha}\left(-\xi^{\alpha}\right)}{2}, \\
\sin _{\alpha}(\xi) & =\frac{E_{\alpha}\left(i \xi^{\alpha}\right)-E_{\alpha}\left(-i \xi^{\alpha}\right)}{2 i}, \\
\cos _{\alpha}(\xi) & =\frac{E_{\alpha}\left(i \xi^{\alpha}\right)+E_{\alpha}\left(-i \xi^{\alpha}\right)}{2 i}, \\
\tanh _{\alpha}(\xi) & =\frac{\sinh _{\alpha}(\xi)}{\cosh _{\alpha}(\xi)}, \quad \operatorname{coth}_{\alpha}(\xi)=\frac{\cosh _{\alpha}(\xi)}{\sinh _{\alpha}(\xi)}, \\
\operatorname{sech}_{\alpha}(\xi) & =\frac{1}{\cosh _{\alpha}(\xi)}, \quad \operatorname{csch}_{\alpha}(\xi)=\frac{1}{\sinh _{\alpha}(\xi)}, \\
\tan _{\alpha}(\xi) & =\frac{\sin _{\alpha}(\xi)}{\cos _{\alpha}(\xi)}, \quad \cot _{\alpha}(\xi)=\frac{\cos _{\alpha}(\xi)}{\sin _{\alpha}(\xi)}, \\
\sec _{\alpha}(\xi) & =\frac{1}{\cos _{\alpha}(\xi)}, \quad \csc _{\alpha}(\xi)=\frac{1}{\sin _{\alpha}(\xi)} .
\end{aligned}
$$

Step 3. Substituting (8) into (7) and using (9), collecting all terms with the same order of $F(\xi)$ together, and then setting coefficients of the polynomial to zero yield a set of algebraic equations for $a_{0}, a_{j}, b_{j}(j=1,2, \ldots, n)$, and $\omega$.

Step 4. Solving the algebraic equations obtained in Step 3, the constants $a_{0}, a_{j}, b_{j}(j=1,2, \ldots, n)$, and $\omega$ can be expressed. Substituting these values into expression (8), we can obtain the general form of the exact solution of (7).

Step 5. Substituting the exact solutions of (9) into the general form of exact solution obtained in Step 4, then we can obtain the exact solutions of (5).

Remark 1. If we take $A=\sigma, B=0$, and $C=1$, this agrees with the results obtained by S. Zhang and H.-Q. Zhang [15].

\section{Exact Solutions of (1)}

In this section, we use the above extended fractional Riccati expansion method to explore the exact solutions of time fractional Burgers equation (1). The study of Burgers equation is important since it arises in the approximate theory of flow through a shock wave propagating in a viscous fluid [24] and in the modeling of turbulence [25]. In [26], Inc used the variational iteration method to obtain the numerical solutions of (1) for $\varepsilon=1$. The approximate solution of (1) for $\varepsilon=1$ based on Von-Neumann method is recently considered by ElDanaf and Hadhoud [27]. Bekir et al. [19] solved (1) using the exponential function method based on fractional complex transform to convert fractional differential equations into ordinary differential equations.

Let

$$
u(x, t)=U(\xi), \quad \xi=x+\omega t
$$

where $\omega$ is nonzero constant, and substituting (23) into (1), we obtain

$$
\omega^{\alpha} D_{\xi}^{\alpha} U+\varepsilon U U^{\prime}-\nu U^{\prime \prime}=0 .
$$

Thus, the solution of (24) has the form

$$
U=a_{0}+a_{1} F(\xi)+\frac{b_{1}}{F(\xi)} .
$$

Substituting (25) into (24) and using (9), collecting the coefficients of $F(\xi)$, and then setting the coefficients of $F(\xi)$ to zero, we can obtain a set of algebraic equations about $a_{0}$, $a_{1}, b_{1}, \omega$. Solving the algebraic equations obtained above, we can have the following solutions.

Type 1. When $B=0$, we have the following cases.

Case 1. We have

$$
a_{0}=-\frac{\omega^{\alpha}}{\varepsilon}, \quad a_{1}=\frac{2 \nu C}{\varepsilon}, \quad b_{1}=0,
$$

where $\omega$ is nonzero constants.

Substituting (26) into (25), then according to (10)-(16), we obtain the following hyperbolic function and trigonometric function solutions of (1), respectively. Consider

$$
u_{1}(x, t)=-\frac{\omega^{\alpha}}{\varepsilon}-\frac{2 \nu C}{\varepsilon} \sqrt{-\frac{A}{C}} \tanh _{\alpha}\left(C \sqrt{-\frac{A}{C} \xi}\right)
$$

where $A / C<0, \xi=x+\omega t$.

The profile for exact solution $u_{1}(x, t)$ of (27) is shown in Figure 1. Consider

$$
u_{2}(x, t)=-\frac{\omega^{\alpha}}{\varepsilon}-\frac{2 \nu C}{\varepsilon} \sqrt{-\frac{A}{C}} \operatorname{coth}_{\alpha}\left(C \sqrt{-\frac{A}{C} \xi}\right),
$$

where $A / C<0, \xi=x+\omega t$. Consider

$$
\begin{array}{r}
u_{3}(x, t)=-\frac{\omega^{\alpha}}{\varepsilon}-\frac{2 \nu C}{\varepsilon} \sqrt{-\frac{A}{C}}\left[\operatorname{coth}_{\alpha}\left(2 C \sqrt{-\frac{A}{C} \xi}\right)\right. \\
\left. \pm \operatorname{csch}_{\alpha}\left(2 C \sqrt{-\frac{A}{C}} \xi\right)\right],
\end{array}
$$




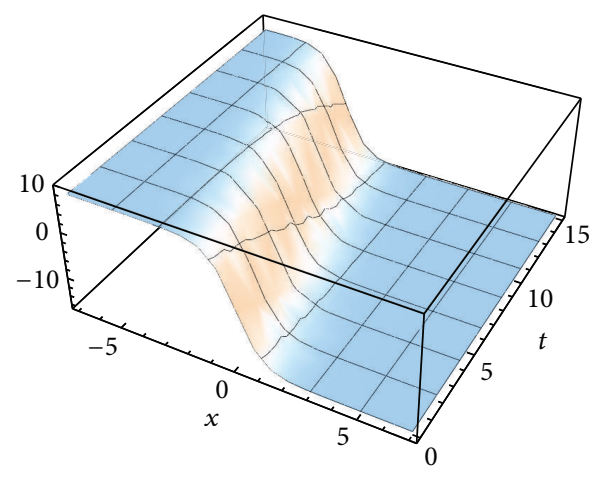

(a) $\alpha=0.5$

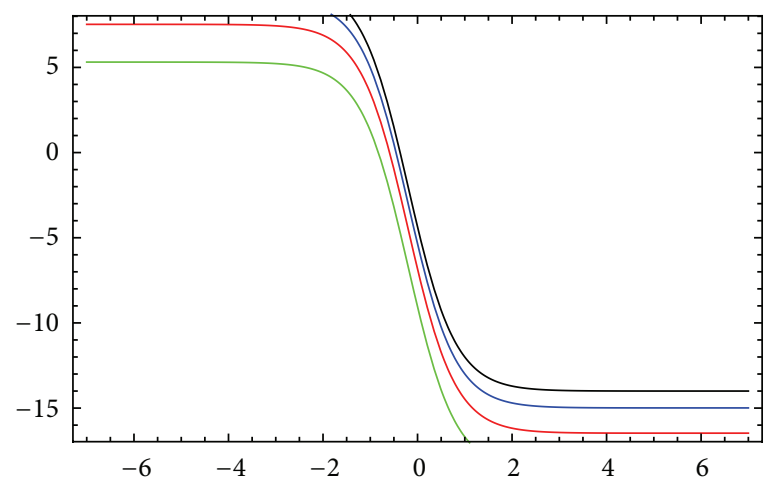

(b) $\alpha=0.25, \alpha=0.5, \alpha=0.75$, and $\alpha=1$

FIgURE 1: The solution $u_{1}(x, t)$ in (27) shows the $3 \mathrm{D}$ and $2 \mathrm{D}$ graphs for the given parameters $A=-1, C=1, \nu=0.6, \varepsilon=0.1$, and $\omega=0.2$.

where $A / C<0, \xi=x+\omega t$. Consider

$$
u_{4}(x, t)=-\frac{\omega^{\alpha}}{\varepsilon}+\frac{2 \nu C}{\varepsilon} \sqrt{\frac{A}{C}} \tan _{\alpha}\left(C \sqrt{\frac{A}{C}} \xi\right)
$$

where $A / C>0, \xi=x+\omega t$. Consider

$$
u_{5}(x, t)=-\frac{\omega^{\alpha}}{\varepsilon}-\frac{2 \nu C}{\varepsilon} \sqrt{\frac{A}{C}} \cot _{\alpha}\left(C \sqrt{\frac{A}{C}} \xi\right)
$$

where $A / C>0, \xi=x+\omega t$. Consider

$$
\begin{aligned}
u_{6}(x, t)=-\frac{\omega^{\alpha}}{\varepsilon}+\frac{2 \nu C}{\varepsilon} \sqrt{\frac{A}{C}}[ & \tan _{\alpha}\left(2 C \sqrt{\frac{A}{C}} \xi\right) \\
& \left. \pm \sec _{\alpha}\left(2 C \sqrt{\frac{A}{C}} \xi\right)\right],
\end{aligned}
$$

where $A / C>0, \xi=x+\omega t$. Consider

$$
\begin{aligned}
u_{7}(x, t)=-\frac{\omega^{\alpha}}{\varepsilon}-\frac{2 \nu C}{\varepsilon} \sqrt{\frac{A}{C}} & {\left[\cot _{\alpha}\left(2 C \sqrt{\frac{A}{C} \xi}\right)\right.} \\
& \left. \pm \csc _{\alpha}\left(2 C \sqrt{\frac{A}{C}} \xi\right)\right]
\end{aligned}
$$

where $A / C>0, \xi=x+\omega t$.

Case 2. We have

$$
a_{0}=-\frac{\omega^{\alpha}}{\varepsilon}, \quad a_{1}=0, \quad b_{1}=-\frac{2 v A}{\varepsilon},
$$

Substituting (34) into (25), then according to (12), (15), and (16), we obtain the following hyperbolic function and trigonometric function solutions of (1), respectively:

$$
\begin{aligned}
u_{8}(x, t)= & \left(2 \nu A \sinh _{\alpha}\left(2 C \sqrt{-\frac{A}{C}} \xi\right)\right. \\
& \left.-\omega^{\alpha} \sqrt{-\frac{A}{C}} \cosh _{\alpha}\left(2 C \sqrt{-\frac{A}{C}} \xi\right) \mp \omega^{\alpha} \sqrt{-\frac{A}{C}}\right) \\
& \times\left(\varepsilon \sqrt{-\frac{A}{C}}\left[\cosh _{\alpha}\left(2 C \sqrt{-\frac{A}{C}} \xi\right) \pm 1\right]\right)^{-1},
\end{aligned}
$$

where $A / C<0, \xi=x+\omega t$. Consider

$$
\begin{aligned}
u_{9}(x, t)=-( & \alpha \sqrt{\frac{A}{C}} \sin _{\alpha}\left(2 C \sqrt{\frac{A}{C}} \xi\right) \\
& \left.+2 \nu A \cos _{\alpha}\left(2 C \sqrt{\frac{A}{C}} \xi\right) \pm \omega^{\alpha} \sqrt{\frac{A}{C}}\right)
\end{aligned}
$$

$$
\times\left(\varepsilon \sqrt{\frac{A}{C}}\left[\sin _{\alpha}\left(2 C \sqrt{\frac{A}{C}} \xi\right) \pm 1\right]\right)^{-1},
$$

where $A / C>0, \xi=x+\omega t$. Consider

$$
\begin{aligned}
u_{10}(x, t)= & \left(2 \nu A \sin _{\alpha}\left(2 C \sqrt{\frac{A}{C}} \xi\right)\right. \\
& \left.-\omega^{\alpha} \sqrt{\frac{A}{C}} \cos _{\alpha}\left(2 C \sqrt{\frac{A}{C}} \xi\right) \mp \omega^{\alpha} \sqrt{\frac{A}{C}}\right) \\
& \times\left(\varepsilon \sqrt{\frac{A}{C}}\left[\cos _{\alpha}\left(2 C \sqrt{\frac{A}{C}} \xi\right) \pm 1\right]\right)^{-1},
\end{aligned}
$$

where $A / C>0, \xi=x+\omega t$. 


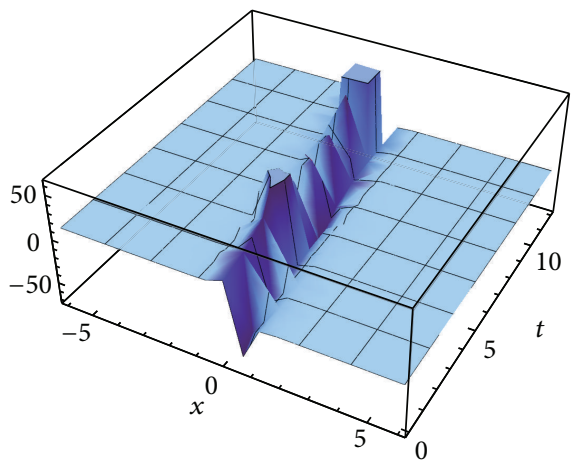

(a) $\alpha=0.4$

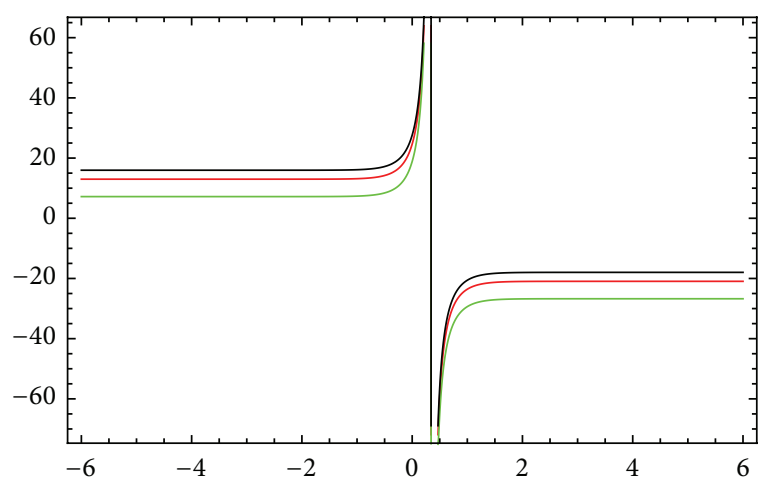

(b) $\alpha=0.01, \alpha=0.4$, and $\alpha=1$

Figure 2: The solution $u_{14}(x, t)$ in (44) shows the 3D and 2D graphs for the given parameters $A=-1, B=2, C=1, v=0.6, \varepsilon=0.1$, and $\omega=0.1$.

Case 3. We have

$$
a_{0}=-\frac{\omega^{\alpha}}{\varepsilon}, \quad a_{1}=\frac{2 \nu C}{\varepsilon}, \quad b_{1}=-\frac{2 \nu A}{\varepsilon},
$$

where $\omega$ is nonzero constants.

Substituting (38) into (25), then according to (10) and (13), we obtain the following hyperbolic function and trigonometric function solutions of (1), respectively:

$$
\begin{aligned}
u_{11}(x, t)= & -\frac{\omega^{\alpha}}{\varepsilon}-\frac{2 \nu C}{\varepsilon} \sqrt{-\frac{A}{C}} \tanh _{\alpha}\left(C \sqrt{-\frac{A}{C}} \xi\right) \\
& +\frac{2 \nu A}{\varepsilon \sqrt{-A / C}} \operatorname{coth}_{\alpha}\left(C \sqrt{-\frac{A}{C}} \xi\right)
\end{aligned}
$$

where $A / C<0, \xi=x+\omega t$. Consider

$$
\begin{aligned}
u_{12}(x, t)= & -\frac{\omega^{\alpha}}{\varepsilon}+\frac{2 \nu C}{\varepsilon} \sqrt{\frac{A}{C}} \tan _{\alpha}\left(C \sqrt{\frac{A}{C}} \xi\right) \\
& -\frac{2 \nu A}{\varepsilon \sqrt{A / C}} \cot _{\alpha}\left(C \sqrt{\frac{A}{C}} \xi\right),
\end{aligned}
$$

where $A / C>0, \xi=x+\omega t$.

Type 2. When $A=0$ and $B C \neq 0$, we have

$$
a_{0}=\frac{\nu B-\omega^{\alpha}}{\varepsilon}, \quad a_{1}=\frac{2 \nu C}{\varepsilon}, \quad b_{1}=0,
$$

where $\omega$ is nonzero constants.

Substituting (41) into (25), then according to (17), we obtain the following hyperbolic function solution of (1):

$$
\begin{aligned}
u_{13}(x, t)= & \frac{v B-\omega^{\alpha}}{\varepsilon} \\
& -\frac{2 \nu B d}{\varepsilon\left[d+\cosh _{\alpha}(B \xi)-\sinh _{\alpha}(B d)\right]},
\end{aligned}
$$

where $\xi=x+\omega t$
Type 3. When $A B C \neq 0$, we have

$$
a_{0}=\frac{\nu B-\omega^{\alpha}}{\varepsilon}, \quad a_{1}=\frac{2 \nu C}{\varepsilon}, \quad b_{1}=0,
$$

where $\omega$ is nonzero constants.

Substituting (43) into (25), then according to (18)-(21), we obtain the following hyperbolic function and trigonometric function solutions of (1), respectively:

$$
\begin{aligned}
u_{14}(x, t)= & \frac{\nu B-\omega^{\alpha}}{\varepsilon} \\
& +\left(4 \nu C A \cosh _{\alpha}\left(\left(\frac{\sqrt{\Delta}}{2}\right) \xi\right)\right) \\
& \times\left(\varepsilon \left[\sqrt{\Delta} \sinh _{\alpha}\left(\left(\frac{\sqrt{\Delta}}{2}\right) \xi\right)\right.\right. \\
& \left.\left.-B \cosh _{\alpha}\left(\left(\frac{\sqrt{\Delta}}{2}\right) \xi\right)\right]\right)^{-1},
\end{aligned}
$$

where $\Delta=B^{2}-4 A C>0, \xi=x+\omega t$.

The profile for exact solution $u_{14}(x, t)$ of (44) is shown in Figure 2. Consider

$$
\begin{aligned}
u_{15}(x, t)= & \frac{\nu B-\omega^{\alpha}}{\varepsilon} \\
& -\left(4 \nu C A \sinh _{\alpha}\left(\left(\frac{\sqrt{\Delta}}{2}\right) \xi\right)\right) \\
& \times\left(\varepsilon \left[B \sinh _{\alpha}\left(\left(\frac{\sqrt{\Delta}}{2}\right) \xi\right)\right.\right. \\
& \left.\left.\quad-\sqrt{\Delta} \cosh _{\alpha}\left(\left(\frac{\sqrt{\Delta}}{2}\right) \xi\right)\right]\right)^{-1},
\end{aligned}
$$




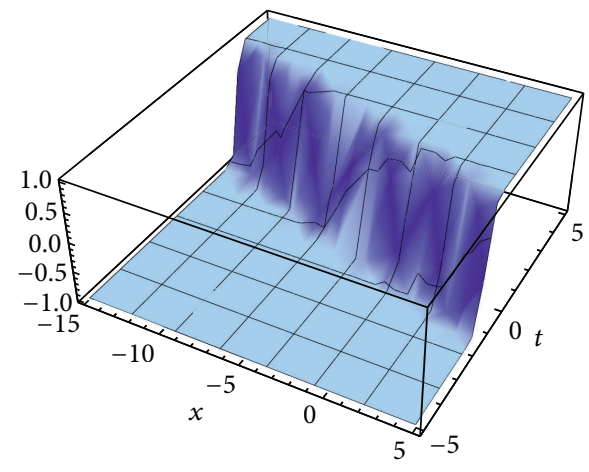

(a) $\alpha=0.5$

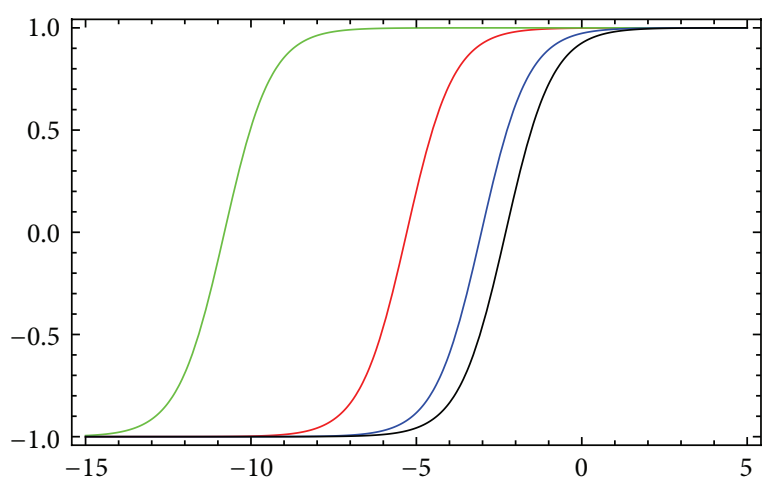

(b) $\alpha=0.35, \alpha=0.5, \alpha=0.75$, and $\alpha=1$

Figure 3: The solution $u_{4}(x, t)$ in (56) shows the 3D and 2D graphs for the given parameter $\gamma=2.3$.

where $\Delta=B^{2}-4 A C>0, \xi=x+\omega t$. Consider

$$
\begin{aligned}
u_{16}(x, t)= & \frac{\nu B-\omega^{\alpha}}{\varepsilon} \\
& -\left(4 \nu C A \cos _{\alpha}\left(\left(\frac{\sqrt{-\Delta}}{2}\right) \xi\right)\right) \\
& \times\left(\varepsilon \left[\sqrt{-\Delta} \sin _{\alpha}\left(\left(\frac{\sqrt{-\Delta}}{2}\right) \xi\right)\right.\right. \\
& \left.\left.+B \cos _{\alpha}\left(\left(\frac{\sqrt{-\Delta}}{2}\right) \xi\right)\right]\right)^{-1},
\end{aligned}
$$

where $\Delta=B^{2}-4 A C<0, \xi=x+\omega t$. Consider

$$
\begin{aligned}
u_{17}(x, t)= & \frac{\nu B-\omega^{\alpha}}{\varepsilon} \\
& -\left(4 \nu C A \sin _{\alpha}\left(\left(\frac{\sqrt{-\Delta}}{2}\right) \xi\right)\right) \\
& \times\left(\varepsilon \left[B \sin _{\alpha}\left(\left(\frac{\sqrt{-\Delta}}{2}\right) \xi\right)\right.\right. \\
& \left.\left.-\sqrt{-\Delta} \cos _{\alpha}\left(\left(\frac{\sqrt{-\Delta}}{2}\right) \xi\right)\right]\right)^{-1},
\end{aligned}
$$

where $\Delta=B^{2}-4 A C<0, \xi=x+\omega t$.

\section{Exact Solutions of (2)}

In this section, we use the above extended fractional Riccati expansion method to explore the exact solutions of CahnHilliard equation (2). In the case of $\alpha=1$, this equation is related to a number of interesting physical phenomena like the spinodal decomposition, phase separation, and phase ordering dynamics [19]. The study of Cahn-Hilliard equation is important since it arises in material sciences [28, 29]. However we notice that this equation is very difficult to be solved and several articles investigated it $[19,30]$. Jafari et al. [31] used the fractional subequation method to calculate the numerical solutions of (2). Bekir et al. [19] solved (2) using the exponential function method based on fractional complex transform to convert fractional differential equations into ordinary differential equations.

Let

$$
u(x, t)=U(\xi), \quad \xi=x+\omega t
$$

where $\omega$ is nonzero constant, and substituting (48) into (2), we obtain

$$
\left(\omega^{\alpha}-\gamma\right) D_{\xi}^{\alpha} U-6 U\left(D_{\xi}^{\alpha} U\right)^{2}-\left(3 U^{2}-1\right) D_{\xi}^{2 \alpha} U+D_{\xi}^{4 \alpha} U=0 .
$$

Thus, the solution of (49) has the form

$$
U=a_{0}+a_{1} F(\xi)+\frac{b_{1}}{F(\xi)} .
$$

Substituting (50) into (49) and using (9), collecting the coefficients of $F(\xi)$, and then setting the coefficients of $F(\xi)$ to zero, we can obtain a set of algebraic equations about $a_{0}$, $a_{1}, b_{1}, \omega$. Solving the algebraic equations obtained above, we can have the following solutions.

Type 1. When $B=0$, we have the following cases.

Case 1. We have

$$
\begin{gathered}
a_{0}=0, \quad a_{1}=\frac{\sqrt{2}}{2 A}, \quad b_{1}=0, \\
\gamma=\omega^{\alpha}, \quad C=-\frac{1}{2 A} .
\end{gathered}
$$

Substituting (51) into (50), then according to (10)-(12), we obtain the following hyperbolic function solutions of (2), respectively:

$$
u_{1}(x, t)=\tanh _{\alpha}\left(\frac{\sqrt{2}}{2} \xi\right)
$$

where $\xi=x+\omega t, \gamma=\omega^{\alpha}$. Consider

$$
u_{2}(x, t)=\operatorname{coth}_{\alpha}\left(\frac{\sqrt{2}}{2} \xi\right),
$$




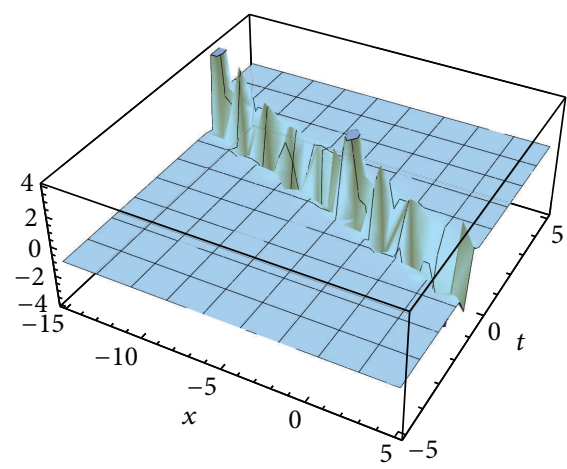

(a) $\alpha=0.4$

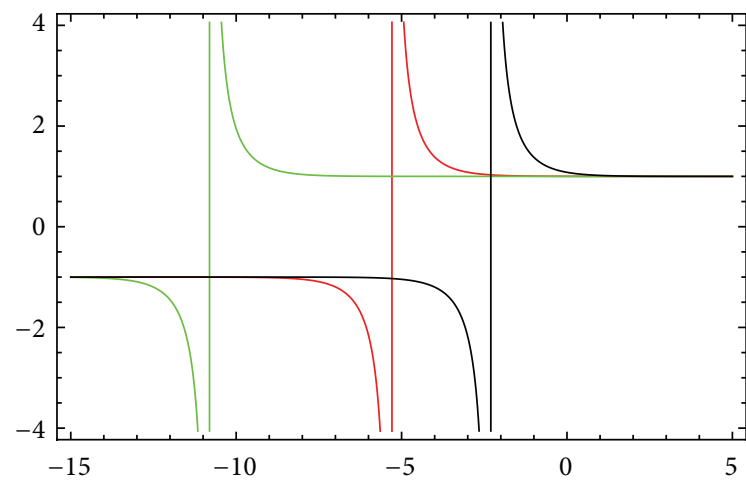

(b) $\alpha=0.01, \alpha=0.4$, and $\alpha=1$

FIgURE 4: The solution $u_{5}(x, t)$ in (58) shows the 3D and 2D graphs for the given parameter $\gamma=2.3$.

where $\xi=x+\omega t$ and $\gamma=\omega^{\alpha}$. Consider

$$
u_{3}(x, t)=\operatorname{coth}_{\alpha}(\sqrt{2} \xi) \pm \operatorname{csch}_{\alpha}(\sqrt{2} \xi) \text {, }
$$

where $\xi=x+\omega t, \gamma=\omega^{\alpha}$.

Case 2. We have

$$
\begin{gathered}
a_{0}=0, \quad a_{1}=0, \quad b_{1}=\sqrt{2} A, \\
\gamma=\omega^{\alpha}, \quad C=-\frac{1}{2 A} .
\end{gathered}
$$

Substituting (55) into (50), then according to (12), we obtain the following hyperbolic function solutions of (2):

$$
u_{4}(x, t)=\frac{\sinh _{\alpha}(\sqrt{2} \xi)}{\cosh _{\alpha}(\sqrt{2} \xi) \pm 1}
$$

where $\xi=x+\omega t, \gamma=\omega^{\alpha}$.

The profile for exact solution $u_{4}(x, t)$ of (56) is shown in Figure 3.

Case 3. We have

$$
\begin{gathered}
a_{0}=0, \quad a_{1}=\frac{\sqrt{2}}{8 A}, \quad b_{1}=\sqrt{2} A, \\
\gamma=\omega^{\alpha}, \quad C=-\frac{1}{8 A} .
\end{gathered}
$$

Substituting (57) into (50), then according to (10), we obtain the following hyperbolic function solution of (2):

$$
u_{5}(x, t)=\frac{1}{2} \tanh _{\alpha}\left(\frac{\sqrt{2}}{4} \xi\right)+\frac{1}{2} \operatorname{coth}_{\alpha}\left(\frac{\sqrt{2}}{4} \xi\right),
$$

where $\xi=x+\omega t, \gamma=\omega^{\alpha}$.

The profile for exact solution $u_{5}(x, t)$ of (58) is shown in Figure 4 .

Case 4. We have

$$
\begin{gathered}
a_{0}=0, \quad a_{1}=\frac{\sqrt{2}}{4 A}, \quad b_{1}=\sqrt{2} A, \\
\gamma=\omega^{\alpha}, \quad C=\frac{1}{4 A} .
\end{gathered}
$$

Substituting (59) into (50), then according to (13), (15), and (16), we obtain the following trigonometric function solutions of (2), respectively:

$$
u_{6}(x, t)=\frac{\sqrt{2}}{2} \tan _{\alpha}\left(\frac{1}{2} \xi\right)+\frac{\sqrt{2}}{2} \cot _{\alpha}\left(\frac{1}{2} \xi\right),
$$

where $\xi=x+\omega t, \gamma=\omega^{\alpha}$. Consider

$$
u_{7}(x, t)= \pm \sqrt{2} \sec _{\alpha}(\xi)
$$

where $\xi=x+\omega t, \gamma=\omega^{\alpha}$. Consider

$$
u_{8}(x, t)=\mp \sqrt{2} \csc _{\alpha}(\xi)
$$

where $\xi=x+\omega t, \gamma=\omega^{\alpha}$.

Type 2. When $A=0$ and $B C \neq 0$, we have

$$
\begin{gathered}
a_{0}=-1, \quad a_{1}=\sqrt{2} C, \quad b_{1}=0, \\
\gamma=\omega^{\alpha}, \quad B=-\sqrt{2} .
\end{gathered}
$$

Substituting (63) into (50), then according to (17), we obtain the following hyperbolic function solution of (2):

$$
u_{9}(x, t)=\frac{d-\cosh _{\alpha}(\sqrt{2} \xi)-\sinh _{\alpha}(\sqrt{2} \xi)}{d+\cosh _{\alpha}(\sqrt{2} \xi)+\sinh _{\alpha}(\sqrt{2} \xi)},
$$

where $\xi=x+\omega t, \gamma=\omega^{\alpha}$.

Type 3. When $A B C \neq 0$, we have the following cases.

Case 2. We have

$$
\begin{gathered}
a_{0}=\frac{\sqrt{2}}{2} B, \quad a_{1}=\frac{\sqrt{2}\left(B^{2}-2\right)}{4 A}, \quad b_{1}=0, \\
\gamma=\omega^{\alpha}, \quad C=\frac{B^{2}-2}{4 A} .
\end{gathered}
$$


Substituting (65) into (50), then according to (18) and (19), we obtain the following hyperbolic function solutions of (2), respectively:

$$
u_{10}(x, t)=\frac{B \sinh _{\alpha}((\sqrt{2} / 2) \xi)-\sqrt{2} \cosh _{\alpha}((\sqrt{2} / 2) \xi)}{\sqrt{2} \sinh _{\alpha}((\sqrt{2} / 2) \xi)-B \cosh _{\alpha}((\sqrt{2} / 2) \xi)}
$$

where $\xi=x+\omega t, \gamma=\omega^{\alpha}$, and $\Delta=B^{2}-4 A C=2>0$. Consider

$$
u_{11}(x, t)=\frac{\sqrt{2} \sinh _{\alpha}((\sqrt{2} / 2) \xi)-B \cosh _{\alpha}((\sqrt{2} / 2) \xi)}{B \sinh _{\alpha}((\sqrt{2} / 2) \xi)-\sqrt{2} \cosh _{\alpha}((\sqrt{2} / 2) \xi)},
$$

where $\xi=x+\omega t, \gamma=\omega^{\alpha}$, and $\Delta=B^{2}-4 A C=2>0$.

Case 5. We have

$$
\begin{gathered}
a_{0}=\frac{\sqrt{2}}{2} B, \quad a_{1}=-\frac{\sqrt{2}\left(B^{2}-2\right)}{8 A}, \quad b_{1}=\sqrt{2} A, \\
\gamma=\omega^{\alpha}, \quad C=-\frac{B^{2}-2}{8 A} .
\end{gathered}
$$

Substituting (68) into (50), then according to (18) and (19), we obtain the following hyperbolic function solutions of (2), respectively:

$$
\begin{aligned}
& u_{12}(x, t) \\
& =\frac{\sqrt{2}}{2} B-\frac{\left(\left(B^{2}-2\right) \sqrt{2} / 4\right) \cosh ((\sqrt{\Delta} / 2) \xi)}{\sqrt{\Delta} \sinh _{\alpha}((\sqrt{\Delta} / 2) \xi)-B \cosh \alpha((\sqrt{\Delta} / 2) \xi)} \\
& +\frac{(\sqrt{2} / 2)\left[\sqrt{\Delta} \sinh _{\alpha}((\sqrt{\Delta} / 2) \xi)-B \cosh _{\alpha}((\sqrt{\Delta} / 2) \xi)\right]}{\cosh _{\alpha}((\sqrt{\Delta} / 2) \xi)}
\end{aligned}
$$

where $\xi=x+\omega t, \gamma=\omega^{\alpha}$, and $\Delta=B^{2}-4 A C=\left(3 B^{2} / 2\right)-1>0$. Consider

$$
\begin{aligned}
& u_{13}(x, t) \\
& =\frac{\sqrt{2}}{2} B+\frac{\left(\left(B^{2}-2\right) \sqrt{2} / 4\right) \sinh _{\alpha}((\sqrt{\Delta} / 2) \xi)}{B \sinh _{\alpha}((\sqrt{\Delta} / 2) \xi)-\sqrt{\Delta} \cosh _{\alpha}((\sqrt{\Delta} / 2) \xi)} \\
& -\frac{(\sqrt{2} / 2)\left[B \sinh _{\alpha}((\sqrt{\Delta} / 2) \xi)-\sqrt{\Delta} \cosh _{\alpha}((\sqrt{\Delta} / 2) \xi)\right]}{\sinh _{\alpha}((\sqrt{\Delta} / 2) \xi)}
\end{aligned}
$$

where $\xi=x+\omega t, \gamma=\omega^{\alpha}$, and $\Delta=B^{2}-4 A C=\left(3 B^{2} / 2\right)-1>0$.
Substituting (68) into (50), then according to (20) and (21), we obtain the following trigonometric function solutions of (2), respectively:

$$
\begin{aligned}
& u_{14}(x, t) \\
& =\frac{\sqrt{2}}{2} B+\frac{\left(\left(B^{2}-2\right) \sqrt{2} / 4\right) \cos _{\alpha}((\sqrt{-\Delta} / 2) \xi)}{\sqrt{-\Delta} \sin _{\alpha}((\sqrt{-\Delta} / 2) \xi)+B \cos _{\alpha}((\sqrt{-\Delta} / 2) \xi)} \\
& -\frac{(\sqrt{2} / 2)\left[\sqrt{-\Delta} \sin _{\alpha}((\sqrt{-\Delta} / 2) \xi)+B \cos _{\alpha}((\sqrt{-\Delta} / 2) \xi)\right]}{\cos _{\alpha}((\sqrt{-\Delta} / 2) \xi)},
\end{aligned}
$$

where $\xi=x+\omega t, \gamma=\omega^{\alpha}$, and $\Delta=B^{2}-4 A C=\left(3 B^{2} / 2\right)-1<0$. Consider

$$
\begin{aligned}
& u_{15}(x, t) \\
& =\frac{\sqrt{2}}{2} B+\frac{\left(\left(B^{2}-2\right) \sqrt{2} / 4\right) \sin _{\alpha}((\sqrt{-\Delta} / 2) \xi)}{B \sin _{\alpha}((\sqrt{-\Delta} / 2) \xi)-\sqrt{-\Delta} \cos _{\alpha}((\sqrt{-\Delta} / 2) \xi)} \\
& -\frac{(\sqrt{2} / 2)\left[B \sin _{\alpha}((\sqrt{-\Delta} / 2) \xi)-\sqrt{-\Delta} \cos _{\alpha}((\sqrt{-\Delta} / 2) \xi)\right]}{\sin _{\alpha}((\sqrt{-\Delta} / 2) \xi)}
\end{aligned}
$$

where $\xi=x+\omega t, \gamma=\omega^{\alpha}$, and $\Delta=B^{2}-4 A C=\left(3 B^{2} / 2\right)-1<0$.

\section{Conclusions}

In this paper, we introduced an extended fractional Riccati expansion method and carried it out to obtain more new exact solutions of the time fractional Burgers equation and the space-time fractional Cahn-Hilliard equation. These exact solutions include hyperbolic function solutions and trigonometric function solutions. To the best of our knowledge, the solutions obtained in this Letter have not been reported in literature. Though some exact solutions of the aforementioned two equations have been obtained via the variational iteration method [26], the Von-Neumann method [27], the exponential function method [19], and fractional subequation method [31], they are different from the solutions obtained in this paper. The paper shows that this method is reliable and simple. We will investigate other types of nonlinear fractional differential equations and so on.

\section{Conflict of Interests}

The authors declare that there is no conflict of interests regarding the publication of this paper.

\section{Acknowledgment}

This research was supported by the Natural Science Foundations of Yunnan Province, China (nos. 2011FZ193 and 2013FZ117). 


\section{References}

[1] R. Hilfer, Applications of Fractional Calculus in Physics, World Scientific, River Edge, NJ, USA, 2000.

[2] A. A. Kilbas, H. M. Srivastava, and J. J. Trujillo, Theory and Applications of Fractional Differential Equations, Elsevier, Amsterdam, The Netherlands, 2006.

[3] R. Metzler and J. Klafter, "The random Walk's guide to anomalous diffusion: a fractional dynamics approach," Physics Reports, vol. 339, no. 1, pp. 1-77, 2000.

[4] F. Santamaria, S. Wils, E. D. Schutter, and G. J. Augustine, "Anomalous diffusion in Purkinje cell dendrites caused by spines," Neuron, vol. 52, no. 4, pp. 635-648, 2006.

[5] G.-c. Wu and E. W. M. Lee, "Fractional variational iteration method and its application," Physics Letters A, vol. 374, no. 25, pp. 2506-2509, 2010.

[6] S. Guo and L. Mei, "The fractional variational iteration method using He's polynomials," Physics Letters A, vol. 375, no. 3, pp. 309-313, 2011.

[7] M. Cui, "Compact finite difference method for the fractional diffusion equation," Journal of Computational Physics, vol. 228, no. 20, pp. 7792-7804, 2009.

[8] J.-H. He, S. K. Elagan, and Z. B. Li, "Geometrical explanation of the fractional complex transform and derivative chain rule for fractional calculus," Physics Letters A, vol. 376, no. 4, pp. 257259, 2012.

[9] R. W. Ibrahim, "Fractional complex transforms for fractional differential equations," Advances in Difference Equations, vol. 2012, article 192, 2012.

[10] S. Zhang, Q. A. Zong, D. Liu, and Q. Gao, "A generalized expfunction method for fractional riccati differential equations," Communications in Fractional Calculus, vol. 1, no. 1, pp. 48-51, 2010.

[11] B. Tang, Y. He, L. Wei, and X. Zhang, "A generalized fractional sub-equation method for fractional differential equations with variable coefficients," Physics Letters A, vol. 376, no. 38-39, pp. 2588-2590, 2012.

[12] B. Zheng, " $\left(G^{\prime} / G\right)$-expansion method for solving fractional partial differential equations in the theory of mathematical physics," Communications in Theoretical Physics, vol. 58, no. 5, pp. 623-630, 2012.

[13] A. Akgül, A. Kılıçman, and M. Inc, "Improved ( $\left.G^{\prime} / G\right)$-expansion method for the space and time fractional foam drainage and KdV equations," Abstract and Applied Analysis, vol. 2013, Article ID 414353, 7 pages, 2013.

[14] B. Lu, "The first integral method for some time fractional differential equations," Journal of Mathematical Analysis and Applications, vol. 395, no. 2, pp. 684-693, 2012.

[15] S. Zhang and H.-Q. Zhang, "Fractional sub-equation method and its applications to nonlinear fractional PDEs," Physics Letters A, vol. 375, no. 7, pp. 1069-1073, 2011.

[16] S. Guo, L. Mei, Y. Li, and Y. Sun, "The improved fractional sub-equation method and its applications to the space-time fractional differential equations in fluid mechanics," Physics Letters A, vol. 376, no. 4, pp. 407-411, 2012.

[17] B. Lu, "Bäcklund transformation of fractional Riccati equation and its applications to nonlinear fractional partial differential equations," Physics Letters A, vol. 376, no. 28-29, pp. 2045-2048, 2012.

[18] E. A.-B. Abdel-Salam and E. A. Yousif, "Solution of nonlinear space-time fractional differential equations using the fractional
Riccati expansion method," Mathematical Problems in Engineering, vol. 2013, Article ID 846283, 6 pages, 2013.

[19] A. Bekir, Güner, and A. C. Cevikel, "Fractional complex transform and exp-function methods for fractional differential equations," Abstract and Applied Analysis, vol. 2013, Article ID 426462, 8 pages, 2013.

[20] M. Caputo, "Linear models of dissipation whose Q is almost frequency independent II," Geophysical Journal International, vol. 13, no. 5, pp. 529-539, 1967.

[21] K. M. Kolwankar and A. D. Gangal, "Local fractional FokkerPlanck equation," Physical Review Letters, vol. 80, no. 2, pp. 214217, 1998.

[22] G. Jumarie, "Modified Riemann-Liouville derivative and fractional Taylor series of nondifferentiable functions further results," Computers \& Mathematics with Applications, vol. 51, no. 9-10, pp. 1367-1376, 2006.

[23] G. Jumarie, "Table of some basic fractional calculus formulae derived from a modified Riemann-Liouville derivative for nondifferentiable functions," Applied Mathematics Letters, vol. 22, no. 3, pp. 378-385, 2009.

[24] J. D. Cole, "On a quasi-linear parabolic equation occurring in aerodynamics," Quarterly of Applied Mathematics, vol. 9, no. 3, pp. 225-236, 1951.

[25] J. M. Burger, "A mathematical model illustrating the theory of turbulence," in Advances in Applied Mechanics, vol. 1, pp. 171199, 1948.

[26] M. Inc, "The approximate and exact solutions of the spaceand time-fractional Burgers equations with initial conditions by variational iteration method," Journal of Mathematical Analysis and Applications, vol. 345, no. 1, pp. 476-484, 2008.

[27] T. S. El-Danaf and A. R. Hadhoud, "Parametric spline functions for the solution of the one time fractional Burgers' equation," Applied Mathematical Modelling, vol. 36, no. 10, pp. 4557-4564, 2012.

[28] S. M. Choo, S. K. Chung, and Y. J. Lee, "A conservative difference scheme for the viscous Cahn-Hilliard equation with a nonconstant gradient energy coefficient," Applied Numerical Mathematics, vol. 51, no. 2-3, pp. 207-219, 2004.

[29] M. E. Gurtin, "Generalized Ginzburg-Landau and Cahn-Hilliard equations based on a microforce balance," Physica D, vol. 92, no. 3-4, pp. 178-192, 1996.

[30] J. Kim, "A numerical method for the Cahn-Hilliard equation with a variable mobility," Communications in Nonlinear Science and Numerical Simulation, vol. 12, no. 8, pp. 1560-1571, 2007.

[31] H. Jafari, H. Tajadodi, N. Kadkhoda, and D. Baleanu, "Fractional subequation method for Cahn-Hilliard and KleinGordon equations," Abstract and Applied Analysis, vol. 2013, Article ID 587179, 5 pages, 2013. 


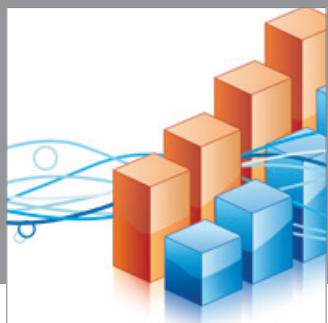

Advances in

Operations Research

mansans

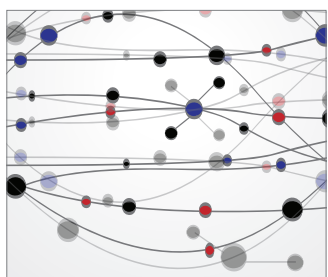

The Scientific World Journal
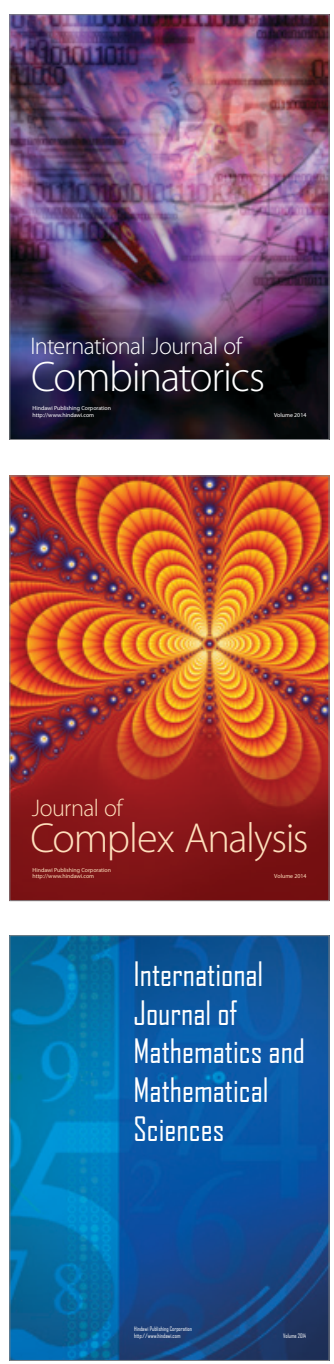
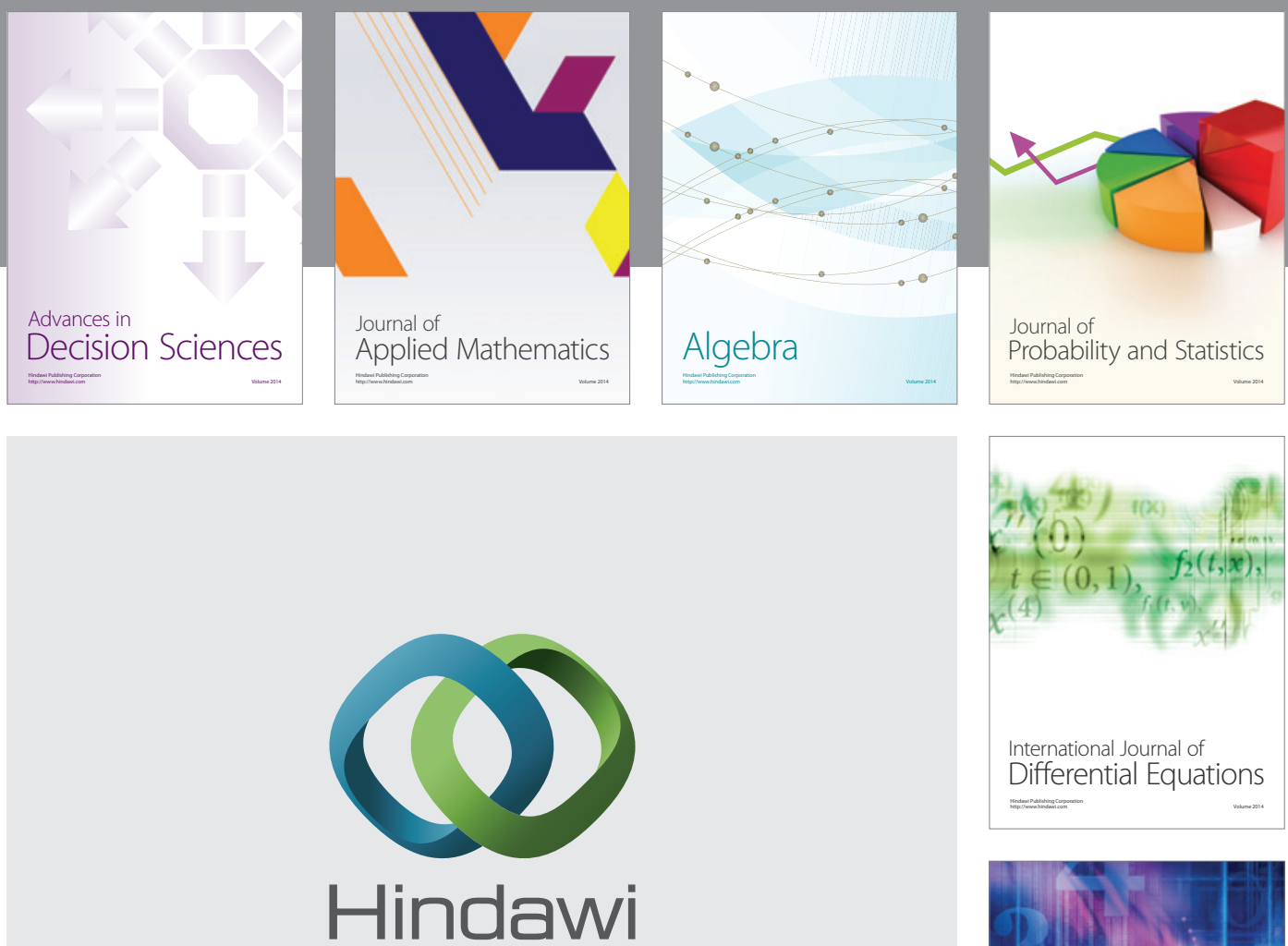

Submit your manuscripts at http://www.hindawi.com
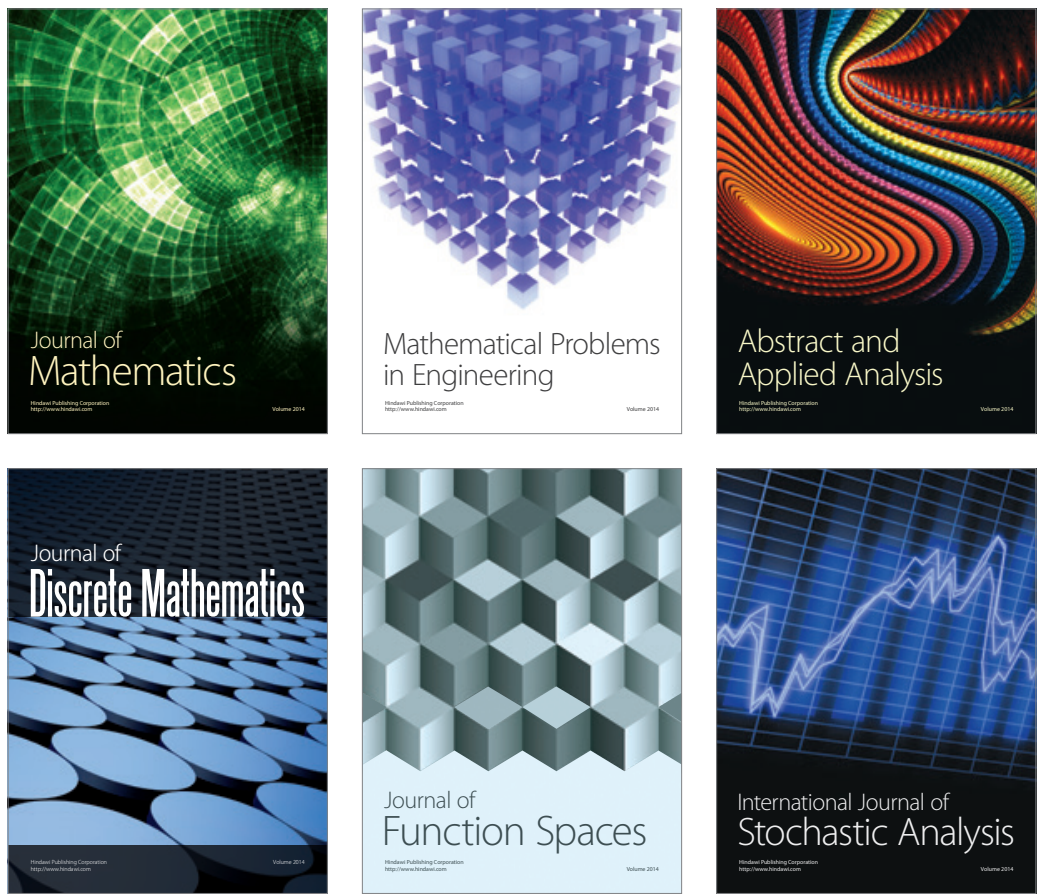

Journal of

Function Spaces

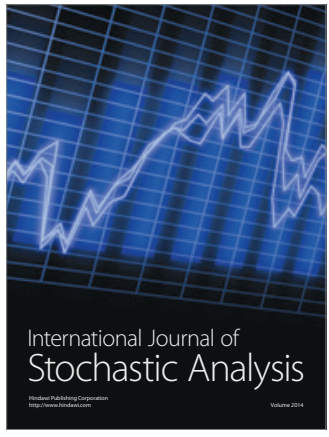

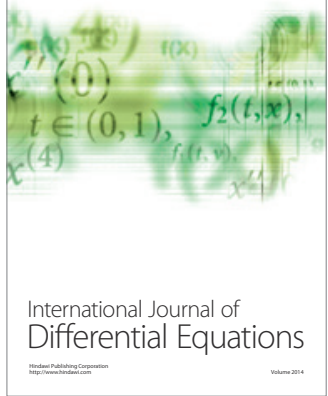
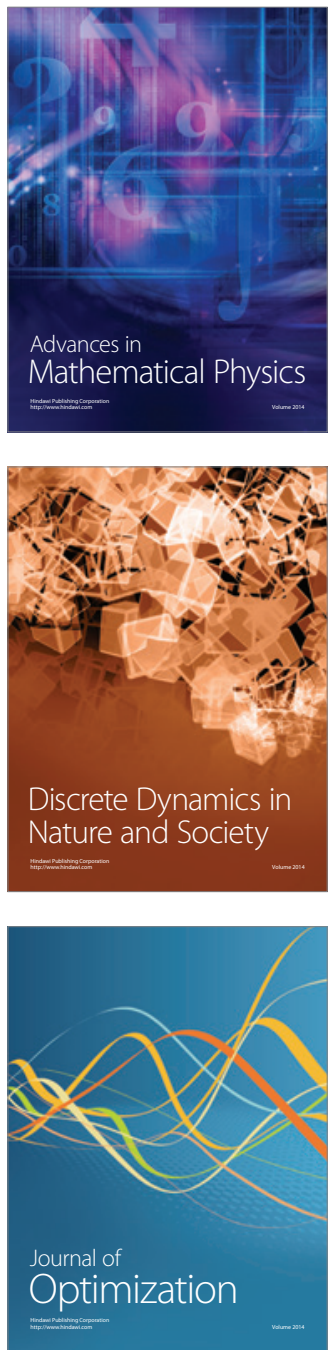